\author{
S. Weyers · G. Selvaggi - S. Monstrey $\cdot$ M. Dhont • \\ R. Van den Broecke - P. De Sutter - G. De Cuypere • \\ G. T' Sjoen $\cdot$ P. Hoebeke
}

\title{
Two-stage versus one-stage sex reassignment surgery in female-to-male transsexual individuals
}

Received: 12 February 2006 / Accepted: 6 May 2006 / Published online: 8 July 2006

(C) Springer-Verlag Berlin / Heidelberg 2006

\begin{abstract}
The objective was to compare the outcome of a combined total hysterectomy-vaginectomy-phalloplasty procedure vs. a vaginectomy-phalloplasty procedure in female-to-male (FTM) gender dysphoric individuals, and to report on a large series of vaginectomies in young women. This was a retrospective study and the setting was the Gender Team at Ghent University Hospital. One hundred and five consecutive cases of vaginectomyphalloplasty with (one-stage) or without (two-stage) total hysterectomy between 1993 and 2003 were included in the study. Patient files of 69 one-stage and of 36 two-stage procedures were reviewed and analysed. Operation time, the need for transfusions, complications, repeated surgery and hospitalisation time were the main outcome measures. Patients were equally distributed over the study period of 10 years. Comparing the two groups, there was a greater need for transfusion in the group of patients undergoing the
\end{abstract}

S. Weyers $(\bowtie) \cdot$ M. Dhont $\cdot$ R. Van den Broecke $\cdot$ P. De Sutter Gender Team, Department of Gynaecology,

Ghent University Hospital,

Ghent, Belgium

e-mail: steven.weyers@ugent.be

Tel.: +32-9-2403793

Fax: +32-9-2403831

G. Selvaggi · S. Monstrey

Gender Team, Department of Plastic and Reconstructive

Surgery, Ghent University Hospital,

Ghent, Belgium

G. De Cuypere

Gender Team, Department of Sexuology and Gender Disorders, Ghent University Hospital,

Ghent, Belgium

G. T. Sjoen

Gender Team, Department of Endocrinology,

Ghent University Hospital,

Ghent, Belgium

P. Hoebeke

Gender Team, Department of Urology,

Ghent University Hospital,

Ghent, Belgium one-stage procedure. There was no difference in operation time, rate of major complications or hospitalisation time. One-stage sex reassignment surgery (SRS) in FTM transsexual individuals is associated with more blood loss. However, there is no difference in operative and postoperative complications. Vaginectomy seems to be a safe and relatively simple procedure in FTM transsexual patients.

Keywords Sex reassignment surgery - Gender dysphoria Hysterectomy

\section{Introduction}

Transsexualism is considered to be the most extreme form of gender identity disorder [1] and typically requires sex reassignment surgery (SRS) following the standards of care of the Harry Benjamin Gender Dysphoria Association [2]. Although not a "sexual" disorder, the term "transsexualism" is still widely used, but the preferred term nowadays is "gender dysphoria" or "gender identity disorder". As early as 1984, Milton T. Edgerton pointed out the importance of multidisciplinary teams in the treatment of gender dysphoria [3]. The goal of SRS in female-to-male (FTM) gender dysphoric patients is removal of the mammary glands and female reproductive organs (uterus, ovaries, tubes and vagina) and construction of a scrotum and a functional phallus. All of these procedures can be performed separately or combined.

The Ghent Gender Team was founded in 1990 and from the beginning consisted of a coordinating psychiatrist, an endocrinologist, a plastic surgeon, a reconstructive urologic surgeon, and a gynaecological surgeon. Since 1990, we have surgically treated over 500 gender dysphoric patients - during the last 2 years at an average of 60 a year. Within such multidisciplinary teams surgery is preceded by extensive counselling by the psychiatrist and hormonal therapy. Gender dysphoric patients will only be allowed to undergo definitive SRS when succeeding in the "real-life test": the patient has to live for at least 1 year under his new opposite sex identity. 
In the first years of our experience (1990-1993) we performed an all-in-one surgical intervention: all of the surgical procedures, including the mastectomy, were included in one single operation. This intervention could take up to $10 \mathrm{~h}$ of anaesthesia time, which resulted in an increased operative risk and substantial blood loss [4]. A few years later we changed our approach and we performed the subcutaneous mastectomy as a separate first step during the real-life test, because this operation greatly facilitates the adjustment to a male life style. In a second stage, usually 612 months after the mastectomy, the hysterectomy, oophorectomy, vaginectomy and phalloplasty were performed. Each of these procedures has its own morbidity and surgical risks, and when combining several steps the risk of morbidity may increase exponentially. Therefore, the question arises if these procedures should all be combined or be performed in separate steps. We conducted a retrospective study comparing the outcome of a combined total hysterectomy-vaginectomy-phalloplasty vs. separate total hysterectomy and vaginectomy-phalloplasty procedures, the hypothesis being that a two-stage approach would lead to less operative morbidity and better surgical outcome. Furthermore, the results of total hysterectomy and vaginectomy in a large group of young women have not been described before.

\section{Materials and methods}

From November 1993 through to April 2003 we performed a total of 105 phalloplasties using the radial forearm flap technique [5]. All of these patients were followed-up by the different members of our gender team and were treated according to the guidelines of the Harry Benjamin International Gender Dysphoria Association [6]. A successful real-life test for at least 2 years and hormonal treatment for at least 12 months were required before SRS. Usually, this treatment consisted of lynestrenol ( $5 \mathrm{mg}$ ), later in association with testosterone. All patients were non-smokers or had stopped smoking before the surgery and hormonal treatment was interrupted 3-4 weeks before the intervention to avoid specific sex steroid-related risks such as thrombosis. All 105 patients had a mastectomy in a separate operation. Phalloplasty was performed in conjunction with total hysterectomy in 69 cases $(65.7 \%)$ while in 36 cases $(34.3 \%)$ the total hysterectomy was conducted separately before the phalloplasty. Most of the patients in the last group were referred to us after the hysterectomy (with or without mastectomy) had been carried out by their own gynaecologist for diverse reasons (bleeding disorders, pain, psychological). In all cases vaginectomy was performed during phalloplasty.

All hysterectomies performed at the time of phalloplasty were done abdominally through a modified low horizontal Pfannenstiel incision (sparing the inferior epigastric vessels). The hysterectomies performed in a separate operation were carried out abdominally or vaginally. The total operation time for the one-stage procedure is calculated from the first incision to the last bandage and includes all procedures: the creation of the flap, the total hysterectomy, the vaginectomy, the creation of the scrotum and the pars fixa of the urethra and the phalloplasty. While calculating the operation time of the two-stage procedure, the time needed for the hysterectomy was not included since this was done previously by another surgeon and no reliable information regarding the operation time of that procedure was available.

When combined with phalloplasty the vaginectomy is started perineally by the urologist by circumcising the hymeneal ring and continuing dissection of the rectovaginal, the vesicovaginal and both paravaginal planes up to the upper third of the vagina. A submucosal vaginectomy is performed. Meanwhile, the gynaecologist starts the hysterectomy in a classical manner with removal of the adnexa, the uterus and the cervix. After transsection of the uterosacral ligaments, the dissection is continued paravaginally until the plane dissected by the urologist is encountered. The uterus with the adnexa, the cervix and the vagina is removed in one piece, subsequently the vesicorectal space is obliterated with two or three stitches. Perineal reconstruction consists of closure of the pelvic floor and approximating the levator ani muscles.

When vaginectomy is performed separately from the hysterectomy, the procedure is performed by the urologist perineally. In all cases the perineal defect is covered with the skin of the labia, thus creating a neo-scrotum. To create the pars fixa of the urethra, the tissue underlying the clitoris is used (Martius flap) [7]. A 18-mm wide strip of mucosa is created and tubularised around an $18 \mathrm{Fr}$ silicone catheter. The clitoris is then transposed to a more ventral position and fixed to the pubic bone (after demucosalisation of the glans). By this manoeuvre the urethra is extended up to the prepubic area where connection with the phallic urethra is made. A second layer using the bulbocavernosus muscle is closed over the reconstructed urethra.

Simultaneously, a team of plastic surgeons prepares the radial forearm flap, which is used for phallic construction [5]. All vaginectomies were performed by the same surgeon $(\mathrm{PH})$.

The files of all 105 patients were reviewed and information on operation time, need for transfusions, complications, repeated surgery and hospitalisation time were noted. The procedures used in this study received ethical approval from the Ethical Committee for Human Experimentations of our hospital (protocol number UZG 2005/227).

Data were analysed for the total group and for both groups separately, group 1 being the group of patients undergoing the hysterectomy together with the phalloplasty (one-stage procedure) and group 2 being the group of patients who underwent a two-stage procedure. Patients were equally distributed over the study period of 10 years.

\section{Results}

The results are presented in Table 1. Average operation time for the whole study was $553 \mathrm{~min}$ (range 390-900). For 
group 1 the average operation time was 557 min (range 390-900), while for group 2 it was 546 min (range 405720 ). Transfusion was needed in 30 individuals $(28.5 \%)$, 24 in group $1(34.7 \%)$ and 6 in group $2(16.7 \%)$, the difference between both groups being statistically significant $(p<0.05)$. Four major complications occurred in group 1: one patient needed urethral reimplantation due to a lesion on the urethra, another patient suffered a pulmonary embolism and two patients had to have a compress that had been left behind during surgery removed within a few weeks. In group 2 no major complications occurred. Thus, the rate of major complications in our series was 3.8\%. There was no statistically significant difference between the two groups. However, a trend of fewer complications in group 2 can be observed, the rate for group 1 being 5.8\% and for group 2 it was $0 \%$.

Haematoma of the perineum occurred in 11 patients $(10.5 \%), 8$ in group $1(11.6 \%)$ and 3 in group $2(8.3 \%)$. Reintervention for perineal haematoma was needed in 6 cases (5.7\%), 3 in group $1(4.3 \%)$ and 3 in group $2(8.3 \%)$. These figures were not significantly different.

Other complications were dehiscence of the inguinal wound in 3 patients, of whom 2 were in group 2, and necrosis of the suprapubic skin in 1 patient belonging to group 2.

There was a non-significant difference in hospitalisation time, number of reinterventions, rate of urinary tract infections, amount of stenosis or fistulas (total, temporary or permanent). In our series $39 \%$ of the patients developed a fistula. This figure is consistent with other data in literature $[8,9]$. Comparing the earlier period of our experience with these techniques (1993-1999) with the more recent years (1999-2003) gives valuable information about the influence of experience of a team on the complication rate.

From 1993 to 1999 we performed SRS in 54 FTM transsexual patients. Four of the 54 patients suffered a severe complication $(7.4 \%)$, a pulmonary embolism, a urethral lesion and two textilomas. The urinary tract complication rate was high, as is generally reported in literature; in the first years of our experience 26 patients (48\%) developed a urinary fistula and $8(15 \%)$ urethral stenosis. With growing experience (51 patients from 1999 up to April 2003) the fistula rate dropped to $29.4 \%$ (15 out of 51) and the stenosis rate to $11.8 \%$ (6 out of 51), but urinary tract complications still occur in more than half of FTM transsexual patients. In this second period no serious complications occurred. The difference in the rate of serious complications between these two periods ( 7.4 vs. $0 \%$ was statistically significant $(p<0.05)$. Textiloma occurred in two patients from group $1(2.9 \%)$ and none from group 2.

\section{Discussion}

Sex reassignment surgery consists of different interventions, all with their own possible complications and risks. All these procedures can be performed separately or combined. Performing the mastectomy as a separate first procedure might facilitate the period of real-life testing for the gender dysphoric individual. Adversely, succeeding with the real-life test can be considered as a conditio sine qua non for commencing surgical transition. The latter view being our standard procedure in the first years of our experience, we combined all steps in a 10 -h procedure. But, as in all combined operations, the total complication rate is not merely the sum of all individual complication rates: the length of these combined procedures, the extensive wound surface, multiple teams working at the same time and the considerable blood loss may create an exponential increase in the complication rate. After 3 years we began to perform the mastectomy as a separate procedure. In about one-third of these transsexual individuals hysterectomy had already been performed upon referral; the other two-thirds of the patients, however, still had an intact female reproductive

Table 1 Hospitalisation time, operation time and summary of complications

\begin{tabular}{|c|c|c|c|c|}
\hline & Total $(n=105)$ & Group $1(n=69)$ & Group $2(n=36)$ & $P$ value group 1 vs. group 2 \\
\hline Hospitalisation (days) & $21(13-44)$ & $21(13-44)$ & $21(13-42)$ & - \\
\hline Operation time (min) & $553(390-900)$ & $557(390-900)$ & $547(405-720)$ & - \\
\hline Serious complications & $4(3.8 \%)$ & $4(5.8 \%)$ & $0(0 \%)$ & 0.18 \\
\hline Reintervention (any reason) & $14(13.3 \%)$ & $9(13 \%)$ & $05(13.8 \%)$ & 0.56 \\
\hline Transfusion needed & $30(28.5 \%)$ & $24(34.7 \%)$ & $6(16.7 \%)$ & 0.05 \\
\hline Textiloma perineal & $2(1.9 \%)$ & $2(2.9 \%)$ & $0(0 \%)$ & 0.43 \\
\hline Haematoma & $11(10.5 \%)$ & $8(11.6 \%)$ & $3(8.3 \%)$ & 0.44 \\
\hline Reintervention for perineal Haematoma & $6(5.7 \%)$ & $3(4.3 \%)$ & $3(8.3 \%)$ & 0.34 \\
\hline Urinary infection & $6(5.7 \%)$ & $3(4.3 \%)$ & $3(8.3 \%)$ & 0.34 \\
\hline Urinary stenosis & $14(13.3 \%)$ & $10(14.5 \%)$ & $4(11.1 \%)$ & 0.44 \\
\hline Total patients with fistula ${ }^{a}$ & $41(39 \%)$ & $28(40.6 \%)$ & $13(36.1 \%)$ & 0.40 \\
\hline Urinary fistula temporary & $26(24.7 \%)$ & $18(26.1 \%)$ & $8(22.2 \%)$ & 0.43 \\
\hline Urinary fistula permanent & $22(20.9 \%)$ & $15(21.7 \%)$ & $7(19.4 \%)$ & 0.50 \\
\hline Inguinal opening & $3(2.8 \%)$ & $2(2.9 \%)$ & $1(2.8 \%)$ & 0.73 \\
\hline Suprapubic skin necrosis & $1(0.9 \%)$ & $1(1.4 \%)$ & $0(0 \%)$ & 0.66 \\
\hline
\end{tabular}

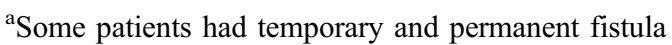


tract. From November 1993 through to April 2003 we performed the vaginectomy and phalloplasty, with or without hysterectomy, in 105 FTM transsexual patients.

There was no statistically significant difference in operation time between groups 1 and 2, notwithstanding the fact that in group 1 hysterectomy was performed during the same procedure and the time needed to perform the hysterectomy was included in the total operation time. This can be explained by the fact that during this one-stage procedure different teams are working at the same time on different sites of the patient-the hysterectomy is performed by the gynaecologist while the team of plastic surgeons create the radial forearm flap and the urologist starts the vaginal part of the vaginectomy.

In our series there was no statistically significant difference between the serious complication rates in groups 1 and 2. In 2 patients from group 1 a compress was left behind during closure of one of the operating sites. During this combined procedure three teams were working on the same patient at the same moment, each team with its own operating nurse, instruments and compresses. This made accurate counting of the compresses on closure more prone to error.

The need for transfusion in this series of 105 patients was $28.5 \%$, with a higher rate in the group of patients who underwent a one-stage operation. This could indicate that the hysterectomy is responsible for a substantial amount of blood loss, although a simple total hysterectomy seldom necessitates a blood transfusion. In this type of operation the largest amount of blood loss is encountered during the vaginectomy, and all patients with a perineal haematoma needed a transfusion. A more reasonable explanation for the difference in the need for transfusion between the two groups is that when performing a vaginectomy with the uterus in place the descending branches of the uterine artery will cause more blood loss compared with a vaginectomy with the uterine artery occluded. Most of the other complications are related to the urethral lengthening and the creation of a penis. SRS is aimed at adjusting the body of the individual to his gender identity. It is generally accepted that SRS helps to alleviate the sequelae of gender dysphoria. In a recent review satisfactory results were reported in $87 \%$ of male-to-female (MTF) and in up to $97 \%$ of FTM transsexual people [10]. These data are comparable to those recently published by our group in that we showed that $80 \%$ of all transsexuals reported improvement of their sexual life after SRS.

The FTM individuals masturbated significantly more frequently than MTF individuals, and a trend towards more sexual satisfaction was seen in the FTM group [11]. Initially, SRS in FTM patients was limited to mastectomy. Nowadays, the gold standard consists of mastectomy, hysterectomy/oophorectomy, vaginectomy and phalloplasty with creation of a scrotum. It is our experience that the vaginectomy is much appreciated by the patients. Despite the high risks involved with this surgery patients still want to have it done. The vagina has a highly symbolic status in female identity and removal of it is of great psychological importance for FTM patients. In the literature little information is available on total vaginectomy. More reports are found on colpocleisis, an intervention in which the vagina is occluded while parts of the vaginal mucosa are spared. Many choose to leave the vagina unchanged in situ or obliterate the vagina with stitches leaving the mucosa behind and leaving a small perineal opening [12, 13]. Leaving the vagina closed in this way often gives rise to complaints of discharge and bad smell. Furthermore, upon sexual excitation vaginal secretion can trickle out of the perineal opening.

Our series of 105 vaginectomies in young patients is the largest series published so far. Our technique of vaginectomy has been proven to be simple and safe. In the total group of 105 patients 5.7\% (6 out of 105) needed reoperation for perineal haematoma. There was a tendency for perineal haematomas to occur more frequently in group 2 , possibly because drainage of blood to the abdomen in these patients was not possible since the peritoneum was not opened.

In one series of 24 patients undergoing total hysterectomy and vaginectomy for prolapsus, the transfusion need was $21 \%$ (5 out of 24 ) and the total complication rate was $37.5 \%$ (9 out of 24) [14]. The rates of major complications in simple abdominal hysterectomy are well known and relatively low. In the VALUE study information was gathered on 37,295 hysterectomies [15]. The rate of major complications in the total abdominal hysterectomy group was $3.6 \%$, major complications being death, deep venous thrombosis, pulmonary embolism, myocardial infarction, renal failure, cerebrovascular accident, septicaemia, necrotising fasciïtis, secondary haemorrhage, fistula, urethral obstruction and visceral damage. We encountered three serious complications ( 3 out of 69 or $4.3 \%$ ), which were undoubtedly related to the hysterectomy-vaginectomy procedure. One patient had a urethral lesion that required urethral reimplantation and two underwent reintervention for a textiloma. When we consider the pulmonary embolism in group 1 as a complication of the hysterectomyvaginectomy procedure, the serious complication rate in the hysterectomy group is $5.8 \%$, which is still in accordance with the results of the VALUE study. Therefore, we can conclude that adding a vaginectomy to the hysterectomy does not increase the risk of having a major complication and that vaginectomy can be considered to be a safe and simple procedure in FTM transsexual patients. We must acknowledge the high incidence of reintervention for perineal haematoma $(4.3 \%)$ in patients undergoing the one-step procedure, but since perineal haematoma can equally be caused by the construction of the neo-scrotum or the phalloplasty we did not consider them to be a consequence of the vaginectomy.

Despite the lack of difference in operation time and major complications, we decided in 2003 to change our policy and perform the SRS in FTM transsexual patients in two steps. Currently, we perform the hysterectomy laparoscopically together with the subcutaneous mastectomy in one procedure and this at least 12 months after the start of hormonal therapy. About 6-12 months later the vaginectomy via the vaginal route is combined with the phalloplasty. Performing 
the SRS in two stages smoothens the transition for the patient and equally facilitates the planning for the surgeons. Since 2003, about 60 FTM transsexual patients have undergone a laparoscopic hysterectomy plus mastectomy and about 35 have undergone all the surgical procedures. The outcome of this series will be dealt with in a future report.

\section{Conclusion}

Gender dysphoria is an incurable condition; however, it is amenable to hormonal and surgical palliation. In FTM gender dysphoric individuals the gold standard in SRS consists of mastectomy, hysterectomy/oophorectomy, vaginectomy and phalloplasty with reconstruction of the fixed part of the urethra. In our series of 105 cases, hysterectomy was performed either simultaneously with the vaginectomy/phalloplasty (group 1) or had been performed previously (group 2). Comparing the two groups, there was a statistically significant difference in the need for transfusion. The significantly higher incidence of blood transfusions in group 1 may result from heavier blood loss from the descending branches of the uterine artery when performing vaginectomy with the uterus still in place.

There was no difference in operation time, rate of major complications or hospitalisation time. The occurrence of fistulas is clearly correlated with the experience acquired. When comparing results of phalloplasties performed within the first 6 years of our experience using the technique of the free radial forearm flap with the results of the following 4 years, the rate of urinary fistulas dropped significantly.

The rate of major complications in simple total abdominal hysterectomy is $3.6 \%$. Since our rate in group 1 was $5.8 \%$, we can conclude that adding vaginectomy to hysterectomy is not associated with a significant increase in major complications. From this large series of vaginectomies, the conclusion can be drawn that vaginectomy seems to be a safe and relatively simple procedure in FTM transsexual patients.

\section{References}

1. Fisk N (1973) Gender dysphoria syndrome (the how, what and why of the disease). In: Laub D, Gandy P (eds) Proceedings of the Second Interdisciplinary Symposium on Gender Dysphoria Syndrome. Stanford University Press, Palo Alto, CA, pp 7-14

2. Meyer W III, Bockting W, Cohen-Kettenis P, Coleman E, Di Ceglie D, Devor H et al (2001) The standards of care for gender identity disorders - sixth version. Int J Transgend http://www. symposion.com/ijt/soc_2001/index.htm.

3. Edgerton MT (1984) The role of surgery in the treatment of transsexualism. Ann Plast Surg 13:473-476

4. Ergeneli MH, Duran EH, Ôzzcan G, Erdogan M (1999) Vaginectomy and laparoscopically assisted vaginal hysterectomy as adjunctive surgery for female-to-male transsexual reassignment: preliminary report. Eur J Obstet Gynecol Reprod Biol 87:35-37

5. Hage J, Bouman F, de Graaf F, Bloem J (1993) Construction of the neophallus in female-to-male transsexuals: the Amsterdam experience. J Urol 149:1463-1468

6. Walker PA, Berger JC, Green R, Laub DR, Reynolds CL, Wollman L (1985) Standards of care: the hormonal and surgical sex reassignment of gender dysphoric persons. Arch Sex Behav 14:79-90

7. Webster G, Sihelnik S, Stone A (1984) Urethrovaginal fistula: a review of the surgical management. J Urol 132:460-462

8. Rohrmann D, Jakse G (2003) Urethroplasty in female-to-male transsexuals. Eur Urol 44:611-614

9. Fang RH, Kao YS, Ma S, Lin JT (1999) Phalloplasty in femaleto-male transsexuals using free radial osteocutaneous flap: a series of 22 cases. Br J Plast Surg 52:217-222

10. Cohen-Kettenis PT, Gooren LJG (1999) Transsexualism: a review of etiology, diagnosis and treatment. J Psychosom Res 46:315-333

11. De Cuypere G, T'Sjoen G, Beerten R, Selvaggi G, De Sutter P, Hoebeke P et al (2005) Sexual and physical health after sex reassignment surgery. Arch Sex Behav 34:679-690

12. Chapin DS (1993) Laparoscopically assisted vaginal hysterectomy in female-to-male transsexuals. Plast Reconstr Surg 91:962

13. Chesson RR, Gilbert DA, Jordan GH, Schlossberg SM, Ramsey GT, Gilbert DM et al (1996) The role of colpocleisis with urethral lengthening in transsexual phalloplasty. Am J Obstet Gynecol 175:1443-1450

14. Smale LE, Smale CL, Mundo NG, Rivera R (1997) Vaginectomy: profile of success in treating vaginal prolapse. Medscape Womens Health 2:5

15. McPherson K, Metcalfe MA, Herbert A, Maresh M, Casbard A, Hargreaves J et al (2004) Severe complications of hysterectomy. The VALUE Study. BJOG 111:688-694 